\title{
$\epsilon$-Henig Saddle Points and Duality of Set-Valued Optimization Problems in Real Linear Spaces
}

\author{
Zhi-Ang Zhou \\ College of Mathematics and Statistics, Chongqing University of Technology, Chongqing 400054, China \\ Correspondence should be addressed to Zhi-Ang Zhou; zhi_ang@163.com
}

Received 8 August 2013; Accepted 5 September 2013

Academic Editors: P. Branchini and V. Kazukauskas

Copyright (c) 2013 Zhi-Ang Zhou. This is an open access article distributed under the Creative Commons Attribution License, which permits unrestricted use, distribution, and reproduction in any medium, provided the original work is properly cited.

\begin{abstract}
We study $\epsilon$-Henig saddle points and duality of set-valued optimization problems in the setting of real linear spaces. Firstly, an equivalent characterization of $\epsilon$-Henig saddle point of the Lagrangian set-valued map is obtained. Secondly, under the assumption of the generalized cone subconvexlikeness of set-valued maps, the relationship between the $\epsilon$-Henig saddle point of the Lagrangian set-valued map and the $\epsilon$-Henig properly efficient element of the set-valued optimization problem is presented. Finally, some duality theorems are given.
\end{abstract}

\section{Introduction}

Saddle points and duality of set-valued optimization, which have close relationship to the generalized convexity of setvalued maps and the efficiency of solutions of set-valued optimization, are two important topics in optimization theory. Recently, many new researches involving saddle points and duality of set-valued optimization have appeared in the literature. Li [1] introduced Benson proper saddle point of the Lagrangian set-valued maps for a set-valued optimization problem in locally convex spaces and established saddle point theorems and duality theorems under the assumption of the cone subconvexlikeness of set-valued maps. Zhao and Rong [2] and Li et al. [3] investigated $\epsilon$-strict saddle points and duality of set-valued optimization problems under the assumption of cone convexlikeness and ic-cone convexlikeness of set-valued maps, respectively. Xia and Qiu [4] obtained saddle point theorems and duality theorems of setvalued optimization problems in the sense of super efficiency under the nearly subconvexlikeness of set-valued maps.

However, in the above mentioned references, saddle points and duality of set-valued optimization were studied in locally convex spaces. How to generalize saddle point theorems and duality theorems of set-valued optimization from locally convex spaces to linear spaces is interesting. Adán and Novo [5] studied saddle points and duality for convexlike vector optimization problems in real linear spaces.
In the $\epsilon$-global prober efficiency, Zhou et al. [6] introduced the concept of the $\epsilon$-global prober saddle point and obtained the relationships between the $\epsilon$-global proper saddle points of Lagrangian set-valued maps and the $\epsilon$-global properly efficient element of set-valued optimization problems.

This paper is a continuation of the research work [7]. The aim of this paper is to investigate $\epsilon$-Henig proper saddle points and duality of set-valued optimization problems in linear spaces. This paper is organized as follows. In Section 2, some preliminaries, including notations and lemmas, are given. In Section 3, we introduce a new notion of $\epsilon$-Henig proper saddle point of the Lagrangian setvalued map in linear spaces and obtain several saddle point theorems. In Section 4, we present three duality theorems including $\epsilon$-weak duality, $\epsilon$-converse duality, and $\epsilon$-strong duality.

\section{Preliminaries}

Let $X$ be a real linear space, and let $Y$ and $Z$ be two real ordered linear spaces. 0 denotes the zero element of every space. Let $K$ be a nonempty subset in $Y$. The generated cone of $K$ is defined as cone $(K):=\{\lambda k \mid k \in K, \lambda \geq 0\} . K$ is called a cone if and only if $\lambda K \subseteq K$ for any $\lambda \geq 0$. A cone $K$ is said to be pointed if and only if $K \cap(-K)=\{0\}$. $K$ is said to be nontrivial if and only if $K \neq\{0\}$ and $K \neq Y$. 
The algebraic dual of $Y$ and $Z$ is denoted by $Y^{*}$ and $Z^{*}$, respectively. Let $C$ and $D$ be two nontrivial pointed convex cones in $Y$ and $Z$, respectively. The algebraic dual cone $C^{+}$and strictly algebraic dual cone $C^{+i}$ of $C$ are, respectively, defined as

$$
\begin{gathered}
C^{+}:=\left\{y^{*} \in Y^{*} \mid\left\langle y, y^{*}\right\rangle \geqslant 0, \forall y \in C\right\}, \\
C^{+i}:=\left\{y^{*} \in Y^{*} \mid\left\langle y, y^{*}\right\rangle>0, \forall y \in C \backslash\{0\}\right\},
\end{gathered}
$$

where $\left\langle y, y^{*}\right\rangle$ denotes the value of the linear functional $y^{*}$ at the point $y$. The meaning of $D^{+}$is similar to that of $C^{+}$.

Definition 1 (see [8]). Let $K$ be a nonempty subset in $Y$. The algebraic interior of $K$ is the set

$$
\begin{array}{r}
\operatorname{cor}(K):=\left\{k \in K \mid \forall h \in Y, \exists \lambda^{\prime}>0,\right. \\
\left.\forall \lambda \in\left[0, \lambda^{\prime}\right], k+\lambda h \in K\right\} .
\end{array}
$$

Definition 2 (see [9]). Let $K$ be a nonempty subset in $Y . K$ is balanced if and only if, for all $x \in K$, for all $\lambda \in[-1,1], \lambda k \in$ $K$. $K$ is called absorbent if and only if $0 \in \operatorname{cor}(K)$.

Definition 3 (see [10]). Let $K$ be a nonempty subset in $Y$. The vector closure of $K$ is the set

$$
\begin{aligned}
\operatorname{vcl}(K):= & \left.\left\{k \in Y \mid \exists h \in Y, \forall \lambda^{\prime}>0, \exists \lambda \in\right] 0, \lambda^{\prime}\right], \\
& k+\lambda h \in K\} .
\end{aligned}
$$

Following Adán and Novo [10], $K$ is vectorially closed ( $v$ closed) if $\operatorname{vcl}(K)=K$.

Definition 4 (see [7]). Let $B$ be a nonempty convex subset in $Y . B$ is a base of $C$ if and only if $C=\operatorname{cone}(B)$ and there exists a balanced, absorbent, and convex set $V$ such that $0 \notin B+V$ in $Y$. Write $C_{V}(B):=\operatorname{cone}(V+B)$.

From now on, we suppose that $\operatorname{cor}(C) \times \operatorname{cor}(D) \neq \emptyset$ and $B$ is a base of $C$. We recall a notion of $\epsilon$-Henig properly efficient point introduced by Zhou et al. [7] in linear spaces.

Definition 5 (see [7]). Let $K \subseteq Y$ and $\epsilon \in C \cdot \bar{y} \in K$ is called an $\epsilon$-Henig properly minimal efficient point with respect to $B$ (denoted by $\bar{y} \in \epsilon-H_{\min }(K, B)$ ) if and only if there exists a balanced, absorbent, and convex set $V$ with $0 \notin B+V$ such that cone $(K-\bar{y}+\epsilon) \cap\left(-C_{V}(B)\right)=\{0\} . \bar{y} \in K$ is called an $\epsilon$-Henig properly maximal efficient point with respect to $B$ (denoted by $\bar{y} \in \epsilon-H_{\max }(K, B)$ ) if and only if there exists a balanced, absorbent, and convex set $V$ with $0 \notin B+V$ such that cone $(K-\bar{y}-\epsilon) \cap C_{V}(B)=\{0\}$.

Let $A$ be a nonempty set in $X$ and $F: A \rightrightarrows Y$ and $G: A \rightrightarrows Z$ be two set-valued maps on $A$. Write $F(A):=\bigcup_{x \in A} F(x),\left\langle F(x), y^{*}\right\rangle:=\left\{\left\langle y, y^{*}\right\rangle \quad \mid \quad y \in\right.$ $F(x)\}$, and $\left\langle F(A), y^{*}\right\rangle:=\bigcup_{x \in A}\left\langle F(x), y^{*}\right\rangle$. The meanings of $G(A),\left\langle G(x), z^{*}\right\rangle$, and $\left\langle G(A), z^{*}\right\rangle$ are similar to those of $F(A),\left\langle F(x), y^{*}\right\rangle$, and $\left\langle F(A), y^{*}\right\rangle$, respectively.

Definition 6 (see [11]). A set-valued map $F: A \rightrightarrows Y$ is called generalized $C$-subconvexlike on $A$ if and only if cone $(F(A))+$ $\operatorname{cor}(C)$ is a convex set in $Y$.
Lemma 7 (see [6]). Let $Z$ be a linear space, and let $M, N \subseteq Z$ be two nonempty sets such that $M-N$ is a convex set in $Z$. If $\operatorname{cor}(M-N) \neq \emptyset$ and $0 \notin \operatorname{vcl}(M-N)$, then there exists $z^{*} \in$ $Z^{*} \backslash\{0\}$ such that $\sup _{z_{2} \in N}\left\langle z_{2}, z^{*}\right\rangle<\inf _{z_{1} \in M}\left\langle z_{1}, z^{*}\right\rangle$.

\section{3. $\epsilon$-Henig Saddle Points}

In this section, we will establish approximate saddle point theorems of set-valued optimization problems in the sense of $\epsilon$-Henig proper efficiency.

Let $F: A \rightrightarrows Y$ and $G: A \rightrightarrows Z$ be two set-valued maps on $A$. We consider the following vector optimization problem with set-valued maps:

$$
\text { (VP) } \min F(x) \text { subject to } G(x) \cap(-D) \neq \emptyset \text {. }
$$

The feasible set of (VP) is defined by $S:=\{x \in A \mid G(x) \cap$ $(-D) \neq \emptyset\}$.

Definition 8 (see [7]). Let $\epsilon \in C . \bar{x} \in S$ is called an $\epsilon$-Henig properly efficient solution of (VP) if and only if there exists $\bar{y} \in F(\bar{x})$ such that $\bar{y} \in \epsilon-H_{\min }(F(S), B)$. The pair $(\bar{x}, \bar{y})$ is called an $\epsilon$-Henig properly efficient element of (VP).

We denote by $L(Z, Y)$ the set of all linear operators from $Z$ to $Y$. A subset $L^{+}(Z, Y)$ of $L(Z, Y)$ is defined as $L^{+}(Z, Y):=$ $\{T \in L(Z, Y) \mid T(D) \subseteq C\}$. The Lagrangian set-valued map of $(\mathrm{VP})$ is defined by

$$
L(x, T):=F(x)+T(G(x)), \quad \forall(x, T) \in A \times L^{+}(Z, Y) .
$$

Consider the following unconstrained vector optimization problem with set-valued maps:

$(\mathrm{UVP})_{T} \min L(x, T)$ subject to $(x, T) \in A \times L^{+}(Z, Y)$.

Lemma 9 (see [7]). Let $\epsilon \in C, \bar{x} \in S$, and $\bar{y} \in F(\bar{x})$. If there exists $\bar{T} \in L^{+}(Z, Y)$ such that $(\bar{x}, \bar{y})$ is an $\epsilon$-Henig properly efficient element of $(U V P)_{\bar{T}}$, then $(\bar{x}, \bar{y})$ is an $\epsilon$-Henig properly efficient element of (VP).

Now, we will introduce a new notion called $\epsilon$-Henig proper saddle point of the Lagrangian set-valued map $L(x, T)$ in linear spaces.

Definition 10. $(\bar{x}, \bar{T}) \in A \times L^{+}(Z, Y)$ is called an $\epsilon$-Henig proper saddle point of the Lagrangian set-valued map $L(x, T)$ if and only if

$$
\begin{aligned}
L(\bar{x}, \bar{T}) \cap \epsilon-H_{\min } & \left(\bigcup_{x \in A} L(x, \bar{T}), B\right) \\
& \cap \epsilon-H_{\max }\left(\bigcup_{T \in L^{+}(Z, Y)} L(\bar{x}, T), B\right) \neq \emptyset .
\end{aligned}
$$

The following proposition is an important equivalent characterization for an $\epsilon$-Henig proper saddle point of the Lagrangian set-valued map $L(x, T)$. 
Proposition 11. Let $D$ be $v$-closed and $\epsilon \in C$. Then, $(\bar{x}, \bar{T}) \in A \times L^{+}(Z, Y)$ is an $\epsilon$-Henig proper saddle point of the Lagrangian set-valued map $L(x, \bar{T})$ if and only if there exist $\bar{y} \in F(\bar{x}), \bar{z} \in G(\bar{x})$, and a balanced, absorbent, and convex set $V$ with $0 \notin B+V$ such that

(i) $\bar{y}+\bar{T}(\bar{z}) \in \epsilon-H_{\min }\left(\bigcup_{x \in A} L(x, \bar{T}), B\right)$;

(ii) $G(\bar{x}) \subseteq-D$;

(iii) $-\bar{T}(\bar{z}) \in C \backslash(\epsilon+C \backslash\{0\})$;

(iv) cone $(F(\bar{x})-\bar{y}-\bar{T}(\bar{z})-\epsilon) \cap C_{V}(B)=\{0\}$.

Proof. Necessity. Let $(\bar{x}, \bar{T})$ be an $\epsilon$-Henig proper saddle point of $L(x, T)$. Then, there exist $\bar{y} \in F(\bar{x})$ and $\bar{z} \in G(\bar{x})$ such that

$$
\begin{gathered}
\bar{y}+\bar{T}(\bar{z}) \in \epsilon-H_{\min }\left(\bigcup_{x \in A} L(x, \bar{T}), B\right), \\
\bar{y}+\bar{T}(\bar{z}) \in \epsilon-H_{\max }\left(\bigcup_{T \in L^{+}(Z, Y)} L(\bar{x}, T), B\right) .
\end{gathered}
$$

Equation (8) shows that (i) holds. By (9), there exists a balanced, absorbent, and convex set $V$ with $0 \notin B+V$ such that

$$
\text { cone }\left(\bigcup_{T \in L^{+}(Z, Y)} L(\bar{x}, T)-\bar{y}-\bar{T}(\bar{z})-\epsilon\right) \cap C_{V}(B)=\{0\} \text {. }
$$

Taking $T=0$ in (10), we obtain

$$
\text { cone }(F(\bar{x})-\bar{y}-\bar{T}(\bar{z})-\epsilon) \cap C_{V}(B)=\{0\} .
$$

Therefore, (iv) holds. Since $\bar{y} \in F(\bar{x})$ and $V$ is absorbent in $Y$, it follows from (11) that

$$
\text { cone }(\bar{T}(\bar{z})+\epsilon) \cap(-B)=\emptyset .
$$

Because cone $(B)=C$, it follows from (12) that cone $(\bar{T}(\bar{z})+$ $\epsilon) \cap(-C \backslash\{0\})=\emptyset$. Clearly, $(\bar{T}(\bar{z})+\epsilon) \cap(-C \backslash\{0\})=\emptyset$. Therefore,

$$
-\bar{T}(\bar{z}) \notin \epsilon+C \backslash\{0\} .
$$

We assert that $-\bar{z} \in D$. Otherwise, by Lemma 7 , it is easy to prove (see the proof of Proposition 4.1 in [6]) that there exists $z_{1}^{*} \in D^{+} \backslash\{0\}$ such that $\left\langle\bar{z}, z_{1}^{*}\right\rangle>0$. Taking $b_{1} \in B$, we define a vector-valued map $T_{1}: Z \rightarrow Y$ as follows:

$$
T_{1}(z)=\frac{\left\langle z, z_{1}^{*}\right\rangle}{\left\langle\bar{z}, z_{1}^{*}\right\rangle}\left(b_{1}+\epsilon\right)+\bar{T}(z), \quad \forall z \in Z .
$$

Clearly, $T_{1} \in L^{+}(Z, Y)$ and $T_{1}(\bar{z})-\bar{T}(\bar{z})-\epsilon=b_{1} \in C_{V}(B)$. On the other hand, $T_{1}(\bar{z})-\bar{T}(\bar{z})-\epsilon \epsilon \operatorname{cone}\left(\bigcup_{T \in L^{+}(Z, Y)} L(\bar{x}, T)-\right.$ $\bar{y}-\bar{T}(\bar{z})-\epsilon)$. Since $0 \notin B+V, b_{1} \neq 0$. Therefore,

$$
\text { cone }\left(\bigcup_{T \in L^{+}(Z, Y)} L(\bar{x}, T)-\bar{y}-\bar{T}(\bar{z})-\epsilon\right) \cap C_{V}(B) \neq\{0\} \text {, }
$$

which contradicts (10). Hence, $-\bar{z} \in D$. Since $\bar{T} \in L^{+}(Z, Y)$, we have

$$
-\bar{T}(\bar{z}) \in C .
$$

It follows from (13) and (16) that (iii) holds. We assert that $G(\bar{x}) \subseteq-D$. Otherwise, there exists $z_{1} \in G(\bar{x})$ such that $z_{1} \notin$ $-D$. Similar to the above proof, there exists $z_{2}^{*} \in D^{+} \backslash\{0\}$ such that $\left\langle z_{1}, z_{2}^{*}\right\rangle>0$. Taking $b_{2} \in B$, we define a vector-valued map $T_{2}: Z \rightarrow Y$ as follows:

$$
T_{2}(z)=\frac{\left\langle z, z_{2}^{*}\right\rangle}{\left\langle z_{1}, z_{2}^{*}\right\rangle}\left(b_{2}+\epsilon\right), \quad \forall z \in Z .
$$

Clearly,

$$
\begin{gathered}
T_{2} \in L^{+}(Z, Y), \\
T_{2}\left(z_{1}\right)-\bar{T}(\bar{z})-\epsilon=b_{2}-\bar{T}(\bar{z}) \in B+C \subseteq C \backslash\{0\} .
\end{gathered}
$$

Since $\bar{y} \in F(\bar{x})$ and $z_{1} \in G(\bar{x})$, it follows from (10) and (18) that

$$
\text { cone }\left(T_{2}\left(z_{1}\right)-\bar{T}(\bar{z})-\epsilon\right) \cap C_{V}(B)=\{0\} .
$$

By (20), it is easy to check that $T_{2}\left(z_{1}\right)-\bar{T}(\bar{z})-\epsilon \notin C \backslash\{0\}$, which contradicts (19). Therefore, (ii) holds.

Sufficiency. Since $\bar{y}+\bar{T}(\bar{z}) \in L(\bar{x}, \bar{T})$, by condition (i), we only prove that

$$
\bar{y}+\bar{T}(\bar{z}) \in \epsilon-H_{\max }\left(\bigcup_{T \in L^{+}(Z, Y)} \mathrm{L}(\bar{x}, T), B\right) .
$$

We assert

$$
\text { cone }\left(\bigcup_{T \in L^{+}(Z, Y)} L(\bar{x}, T)-\bar{y}-\bar{T}(\bar{z})-\epsilon\right) \cap C_{V}(B)=\{0\} \text {. }
$$

Otherwise, there exists $r_{1}>0, r_{2}>0, T_{3} \in L^{+}(Z, Y), y^{\prime} \in$ $F(\bar{x}), z^{\prime} \in G(\bar{x}), v \in V$, and $b_{3} \in B$ such that $r_{1}\left(y^{\prime}+T_{3}\left(z^{\prime}\right)-\right.$ $\bar{y}-\bar{T}(\bar{z})-\epsilon)=r_{2}(v+b)$. Clearly,

$$
r_{1}\left(y^{\prime}-\bar{y}-\bar{T}(\bar{z})-\epsilon\right)=r_{2}\left(v+b_{3}\right)-r_{1} T_{3}\left(z^{\prime}\right) .
$$

By condition (ii), $z^{\prime} \in-D$. Since $T_{3} \in L^{+}(Z, Y),-T_{3}\left(z^{\prime}\right) \in C$. Therefore, there exist $r_{3} \geq 0$ and $b_{4} \in B$ such that

$$
-T_{3}\left(z^{\prime}\right)=r_{3} b_{4} .
$$

It follows from (23) and (24) that

$$
\begin{aligned}
r_{1}\left(y^{\prime}-\right. & \bar{y}-\bar{T}(\bar{z})-\epsilon) \\
= & r_{2}\left(v+b_{3}\right)+r_{1} r_{3} b_{4} \\
= & r_{2} v+\left(r_{2} b_{3}+r_{1} r_{3} b_{4}\right)=\left(r_{2}+r_{1} r_{3}\right) \\
& \times\left[\frac{r_{2}}{r_{2}+r_{1} r_{3}} v+\left(\frac{r_{2}}{r_{2}+r_{1} r_{3}} b_{3}+\frac{r_{1} r_{3}}{r_{2}+r_{1} r_{3}} b_{4}\right)\right] .
\end{aligned}
$$


Since $0<r_{2} /\left(r_{2}+r_{1} r_{3}\right) \leq 1$, it follows from the balance of $V$ that

$$
\frac{r_{2}}{r_{2}+r_{1} r_{3}} v \in V
$$

It follows from the convexity of $B$ that

$$
\frac{r_{2}}{r_{2}+r_{1} r_{3}} b_{3}+\frac{r_{1} r_{3}}{r_{2}+r_{1} r_{3}} b_{4} \in B
$$

Since $0 \notin V+B$ and $r_{2}+r_{1} r_{3}>0$, it follows from (25)-(27) that

$$
\begin{aligned}
0 & \neq r_{1}\left(y^{\prime}-\bar{y}-\bar{T}(\bar{z})-\epsilon\right) \\
& \in \operatorname{cone}(F(\bar{x})-\bar{y}-\bar{T}(\bar{z})-\epsilon) \cap C_{V}(B),
\end{aligned}
$$

which contradicts condition (iv). Therefore, (22) holds. Thus, (21) holds.

Remark 12. According to Theorem 1 in [12], the notion of $\epsilon$ strictly efficient point is equivalent to the notion of $\epsilon$-Henig properly efficient point in locally convex spaces. Moreover, the generalized subconvexlikeness of the set-valued map $F$ is equivalent to ic-cone convexlikeness of the set-valued map $F$ introduced by Sach [13] when the topological interior $\operatorname{int}(C) \neq \emptyset$. Therefore, Proposition 11 generalizes Proposition 5.1 in [3] from locally convex spaces to real linear spaces.

Theorem 13. Let $D$ be $v$-closed and $\epsilon \in C$. If $(\bar{x}, \bar{T}) \in A \times$ $L^{+}(Z, Y)$ is an $\epsilon$-Henig proper saddle point of the Lagrangian set-valued map $L(x, T)$, then there exist $\bar{y} \in F(\bar{x})$ and $\bar{z} \epsilon$ $G(\bar{x})$ such that $(\bar{x}, \bar{y})$ is an $\bar{\epsilon}$-Henig properly efficient element of $(V P)$, where $\bar{\epsilon}=\epsilon-\bar{T}(\bar{z})$.

Proof. Since $(\bar{x}, \bar{T}) \in A \times L^{+}(Z, Y)$ is an $\epsilon$-Henig proper saddle point of the Lagrangian set-valued map $L(x, T)$, it follows from Proposition 11 that there exist $\bar{y} \in F(\bar{x}), \bar{z} \in$ $G(\bar{x})$, and a balanced, absorbent, and convex set $V$ with $0 \notin$ $B+V$ such that conditions (i)-(iv) hold. By condition (ii), $\bar{x} \in S$. By condition (iii), $\bar{\epsilon}=\epsilon-\bar{T}(\bar{z}) \in C+C \backslash(\epsilon+C \backslash\{0\}) \subseteq C$. We assert that $(\bar{x}, \bar{y})$ is an $\bar{\epsilon}$-Henig properly efficient element of (VP). Otherwise, for any balanced, absorbent, and convex set $U$ with $0 \notin B+U$, cone $(F(S)-\bar{y}+\bar{\epsilon}) \cap\left(-C_{U}(B)\right) \neq\{0\}$. Therefore, there exist $r_{1}>0, r_{2}>0, x_{1} \in S, y_{1} \in F(S), b_{1} \in$ $B$, and $u_{1} \in U$ such that

$$
r_{1}\left(y_{1}-\bar{y}+\bar{\epsilon}\right)=-r_{2}\left(b_{1}+u_{1}\right) .
$$

It follows from (29) that

$$
\begin{array}{r}
r_{1}\left(y_{1}+\bar{T}(z)+\epsilon-(\bar{y}+\bar{T}(\bar{z}))\right)=-r_{2}\left(b_{1}+u_{1}\right)+r_{1} \bar{T}(z), \\
\forall z \in G(\bar{x}) \cap(-D) .
\end{array}
$$

Since $\bar{T} \in L^{+}(Z, Y)$, for any $z \in G(\bar{x}) \cap(-D)$, it follows from (30) that there exists $c \in C$ such that

$$
r_{1}\left(y_{1}+\bar{T}(z)+\epsilon-(\bar{y}+\bar{T}(\bar{z}))\right)=-r_{2}\left(b_{1}+u_{1}\right)-r_{1} c .
$$

Because $B$ is a base of $C$, there exist $r_{3} \geq 0$ and $b_{2} \in B$ such that $c=r_{3} b_{2}$. By (31), we obtain

$$
\begin{aligned}
r_{1}\left(y_{1}+\right. & \bar{T}(z)+\epsilon-(\bar{y}+\bar{T}(\bar{z}))) \\
= & -r_{2}\left(b_{1}+u_{1}\right)-r_{1} r_{3} b_{2} \\
= & -\left[r_{2} u_{1}+\left(r_{2} b_{1}+r_{1} r_{3} b_{2}\right)\right] \\
= & -\left(r_{2}+r_{1} r_{3}\right) \\
& \quad \times\left[\frac{r_{2}}{r_{2}+r_{1} r_{3}} u_{1}\right. \\
& \left.\quad+\left(\frac{r_{2}}{r_{2}+r_{1} r_{3}} b_{1}+\frac{r_{1} r_{3}}{r_{2}+r_{1} r_{3}} b_{2}\right)\right] \in-C_{U}(B) .
\end{aligned}
$$

Clearly, $r_{1}\left(y_{1}+\bar{T}(z)+\epsilon-(\bar{y}+\bar{T}(\bar{z}))\right) \neq 0$ for all $z \in G(\bar{x}) \cap(-D)$. Therefore, we obtain

$$
\text { cone }\left(\bigcup_{x \in A} L(x, \bar{T})+\epsilon-(\bar{y}+\bar{T}(\bar{z}))\right) \cap\left(-C_{U}(B)\right) \neq\{0\}
$$

which contradicts $\bar{y}+\bar{T}(\bar{z}) \in \epsilon-H_{\min }\left(\bigcup_{x \in A} L(x, \bar{T}), B\right)$. Hence, $(\bar{x}, \bar{y})$ is an $\bar{\epsilon}$-Henig properly efficient element of (VP).

Remark 14. Comparing Theorem 13 with Theorem 4.1 in [6], the notion of $\epsilon$-global proper efficiency has been replaced by the notion of $\epsilon$-Henig proper efficiency and the condition $0 \epsilon$ $G(\bar{x})$ has been dropped.

In order to obtain sufficient conditions of $\epsilon$-Henig proper saddle point under the assumption of the generalized cone subconvexlikeness, we need the following lemma.

Lemma 15 (see [7]). Let $\epsilon \in C, \bar{x} \in S$, and $0 \in G(\bar{x})$. Suppose that the following conditions hold:

(i) $(\bar{x}, \bar{y})$ is an $\epsilon$-Henig properly efficient element of (VP);

(ii) $\bar{I}(x)$ is generalized $C \times D$-subconvexlike on $A$, where $\bar{I}(x)=(F(x)-\bar{y}+\epsilon) \times G(x) ;$

(iii) $\operatorname{vcl}(\operatorname{cone}(G(A)+D))=Z$.

Then, there exists $\bar{T} \in L^{+}(Z, Y)$ such that $(\bar{x}, \bar{y})$ is an $\epsilon$-Henig properly efficient element of $(U V P)_{\bar{T}}$.

By Lemma 15, we easily obtain the following theorem involving the generalized cone subconvexlikeness of setvalued maps.

Theorem 16. Let $D$ be $v$-closed, $\epsilon \in C, \bar{x} \in S$, and $0 \in G(\bar{x})$. Suppose that the following conditions hold:

(i) $(\bar{x}, \bar{y})$ is an $\epsilon$-Henig properly efficient element of (VP);

(ii) $\bar{I}(x)$ is generalized $C \times D$-subconvexlike on $A$, where $\bar{I}(x)=(F(x)-\bar{y}+\epsilon) \times G(x) ;$ 
(iii) $v c l(\operatorname{cone}(G(A)+D))=Z$;

(iv) $\bar{y} \in \epsilon-H_{\max }\left(\bigcup_{T \in L^{+}(Z, Y)} L(\bar{x}, T), C\right)$.

Then, there exists $\bar{T} \in L^{+}(Z, Y)$ such that $(\bar{x}, \bar{T})$ is an $\epsilon$-Henig proper saddle point of $L$.

\section{4. $\epsilon$-Duality}

In this section, we will give several duality theorems characterized by $\epsilon$-Henig proper efficiency of set-valued optimization problems in linear spaces.

Definition 17. Let $\epsilon \in C$ and let $B$ be a base of $C$. The setvalued map $\Phi: L^{+}(Z, Y) \rightrightarrows Y$, defined by $\Phi(T)=\epsilon$ $H_{\text {min }}\left(\bigcup_{x \in A} L(x, T), B\right)$, is called an $\epsilon$-Henig properly dual map of (VP).

Now, we construct the following duality problem of the primal problem (VP):

$$
\text { (VD) } \max \bigcup_{T \in L^{+}(Z, Y)} \Phi(T)
$$

Definition 18. Let $\epsilon \in C . \bar{y} \in \bigcup_{T \in L^{+}(Z, Y)} \Phi(T)$ is called an $\epsilon$ efficient point of (VD) if and only if

$$
\left(\bigcup_{T \in L^{+}(Z, Y)} \Phi(T)-\bar{y}-\epsilon\right) \cap(C \backslash\{0\})=\emptyset .
$$

Theorem 19 ( $\epsilon$-weak duality). Let $\epsilon \in C, \bar{x} \in S$, and $\bar{y} \epsilon$ $\bigcup_{T \in L^{+}(Z, Y)} \Phi(T)$. Then, $(\bar{y}-F(\bar{x})-\epsilon) \cap(C \backslash\{0\})=\emptyset$.

Proof. Since $\bar{y} \in \bigcup_{T \in L^{+}(Z, Y)} \Phi(T)$, there exists $\bar{T} \in L^{+}(Z, Y)$ such that $\bar{y} \in \Phi(\bar{T})$. Clearly, $\bar{y} \in \epsilon-H_{\min }\left(\bigcup_{x \in A} L(x, \bar{T}), B\right)$. Thus, there exists a balanced, absorbent, and convex set $V$ with $0 \notin B+V$ such that cone $\left(\bigcup_{x \in A} L(x, \bar{T})-\bar{y}+\epsilon\right) \cap$ $\left(-C_{V}(B)\right)=\{0\}$. It is easy to check $C \backslash\{0\} \subseteq C_{V}(B) \backslash\{0\}$. Therefore, $\left(\bar{y}-\bigcup_{x \in A} L(x, \bar{T})-\epsilon\right) \cap(C \backslash\{0\})=\emptyset$. Since $\bar{x} \in S \subseteq A,(\bar{y}-F(\bar{x})-\bar{T}(G(\bar{x}))-\epsilon) \cap(C \backslash\{0\})=\emptyset$. Because $\bar{x} \in S$, there exists $\bar{z} \in G(\bar{x}) \cap(-D)$ such that $(\bar{y}-F(\bar{x})-\bar{T}(\bar{z})-\epsilon) \cap(C \backslash\{0\})=\emptyset$. Since $\bar{T}(\bar{z}) \in C$, it is easy to check that $(\bar{y}-F(\bar{x})-\epsilon) \cap(C \backslash\{0\})=\emptyset$.

Theorem 20 ( $\epsilon$-converse duality). Let $\epsilon \in C$ and $\bar{x} \in S$. If $\bar{y} \in F(\bar{x}) \cap \bigcup_{T \in L^{+}(Z, Y)} \Phi(T)$ and $0 \in G(\bar{x})$, then $(\bar{x}, \bar{y})$ is an $\epsilon$-Henig properly efficient element of (VP) and $\bar{y}$ is $\epsilon$-efficient point of (VD).

Proof. Since $\bar{y} \in F(\bar{x}) \cap \bigcup_{T \in L^{+}(Z, Y)} \Phi(T)$, there exists $\bar{T} \in$ $L^{+}(Z, Y)$ such that $\bar{y} \in \Phi(\bar{T})$. It follows from $0 \in G(\bar{x})$ and the definition of $\Phi$ that $(\bar{x}, \bar{y})$ is an $\epsilon$-Henig properly efficient element of $(\mathrm{UVP})_{\bar{T}}$. According to Lemma $9,(\bar{x}, \bar{y})$ is an $\epsilon$-Henig properly efficient element of (VP). Because $\bar{x} \epsilon$ $S$ and $\bar{y} \in \bigcup_{T \in L^{+}(Z, Y)} \Phi(T)$, using Theorem 19, we have $\left(\bigcup_{T \in L^{+}(Z, Y)} \Phi(T)-F(\bar{x})-\epsilon\right) \cap(C \backslash\{0\})=\emptyset$. Clearly, $\left(\bigcup_{T \in L^{+}(Z, Y)} \Phi(T)-\bar{y}-\epsilon\right) \cap(C \backslash\{0\})=\emptyset$. Therefore, $\bar{y}$ is $\epsilon$-efficient point of (VD).
Theorem 21 ( $\epsilon$-strong duality). Let $\epsilon \in C, \bar{x} \in S$, and $0 \epsilon$ $G(\bar{x})$. Suppose that the following conditions hold:

(i) $(\bar{x}, \bar{y})$ is an $\epsilon$-Henig properly efficient element of $(V P)$;

(ii) $\bar{I}(x)$ is generalized $C \times D$-subconvexlike on $A$, where $\bar{I}(x)=(F(x)-\bar{y}+\epsilon) \times G(x) ;$

(iii) $\operatorname{vcl}(\operatorname{cone}(G(A)+D))=Z$.

Then, $\bar{y}$ is $\epsilon$-efficient point of (VD).

Proof. According to Lemma 15, there exists $\bar{T} \in L^{+}(Z, Y)$ such that $(\bar{x}, \bar{y})$ is an $\epsilon$-Henig properly efficient element of $(\mathrm{UVP})_{\bar{T}}$. Since $0 \in G(\bar{x})$, we have

$$
\bar{y} \in \epsilon-H_{\min }\left(\bigcup_{x \in A} L(x, \bar{T}), B\right)=\Phi(\bar{T}) \subseteq \bigcup_{T \in L^{+}(Z, Y)} \Phi(T) .
$$

Since $\bar{y} \in F(\bar{x})$, it follows from Theorem 19 that $\bar{y}$ is $\epsilon$ efficient point of (VD).

\section{Conclusions}

Based on [7], we introduce the concept of $\epsilon$-Henig saddle point of the set-valued map in linear spaces. The relationships between the $\epsilon$-Henig saddle point of the set-valued map and the $\epsilon$-Henig properly efficient element of the set-valued optimization problem are established. Some duality theorems are obtained in the sense of $\epsilon$-Henig proper efficiency. When $\epsilon$-Henig proper efficiency is replaced by $\epsilon$-super efficiency in linear spaces, whether the conclusions of this paper hold is an interesting topic.

\section{Acknowledgments}

This work was supported by the National Nature Science Foundation of China (11271391), the Natural Science Foundation of Chongqing (CSTC 2011jjA00022), and the Science and Technology Project of Chongqing Municipal Education Commission (KJ130830).

\section{References}

[1] Z. F. Li, "Benson proper efficiency in the vector optimization of set-valued maps," Journal of Optimization Theory and Applications, vol. 98, no. 3, pp. 623-649, 1998.

[2] C. Y. Zhao and W. D. Rong, "Optimality conditions for setvalued optimization in the sense of $\epsilon$-strict efficiency," Operations Research Transactions, vol. 12, no. 4, pp. 10-18, 2008.

[3] T. Li, Y. Xu, and C. Zhu, " $\epsilon$-strictly efficient solutions of vector optimization problems with set-valued maps," AsiaPacific Journal of Operational Research, vol. 24, no. 6, pp. 841854, 2007.

[4] L. Y. Xia and J. H. Qiu, "Super efficiency in vector optimization with nearly subconvexlike set-valued maps," Journal of Optimization Theory and Applications, vol. 136, no. 1, pp. 125-137, 2008.

[5] M. Adán and V. Novo, "Duality and saddle points for convexlike vector optimization problems on real linear spaces," Top, vol.13, no. 2, pp. 343-357, 2005. 
[6] Z.-A. Zhou, X.-M. Yang, and J.-W. Peng, " $\epsilon$-optimality conditions of vector optimization problems with set-valued maps based on the algebraic interior in real linear spaces," Optimization Letters, 2013.

[7] Z.-A. Zhou, X.-M. Yang, and J.-W. Peng, " $\epsilon$-Henig proper efficiency of set-valued optimization problems in real ordered linear spaces," Optimization Letters, 2013.

[8] J. Jahn, Vector Optimization: Theory, Applications and Extensions, Springer, Berlin, Germany, 2nd edition, 2011.

[9] S. Z. Shi, Convex Analysis, Shanghai Science and Technology Press, Shanghai, China, 1990.

[10] M. Adán and V. Novo, "Weak efficiency in vector optimization using a closure of algebraic type under cone-convexlikeness," European Journal of Operational Research, vol. 149, no. 3, pp. 641-653, 2003.

[11] Y. W. Huang and Z. M. Li, "Optimality condition and Lagrangian multipliers of vector optimization with set-valued maps in linear spaces," Operations Research Transactions, vol. 5, no. 1, pp. 63-69, 2001.

[12] J. H. Qiu and S. Y. Zhang, "Strictly efficient points and Henig properly efficient points," Journal of Mathematics, vol. 25, pp. 203-209, 2005.

[13] P. H. Sach, "New generalized convexity notion for set-valued maps and application to vector optimization," Journal of Optimization Theory and Applications, vol. 125, no. 1, pp. 157-179, 2005. 


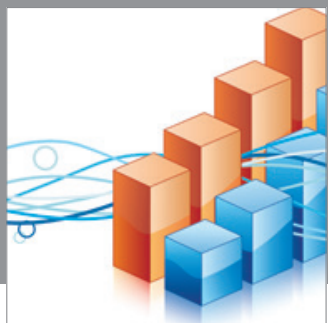

Advances in

Operations Research

mansans

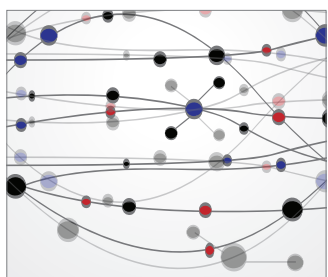

The Scientific World Journal
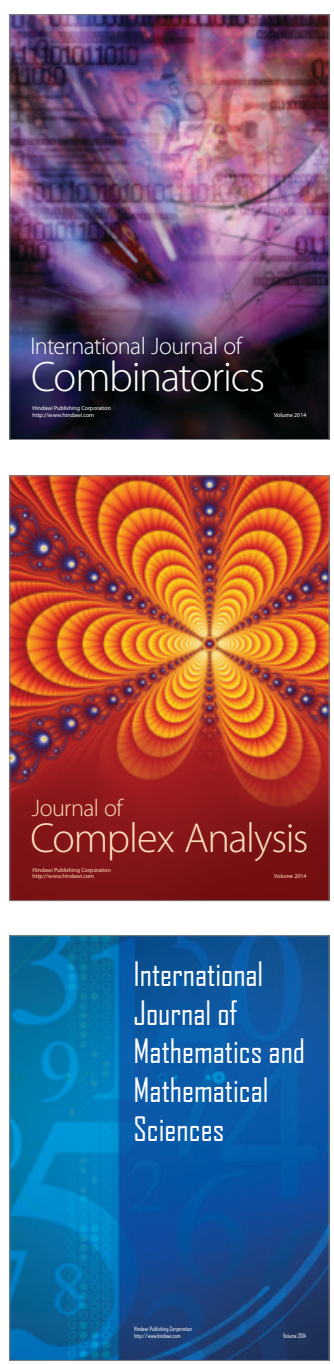
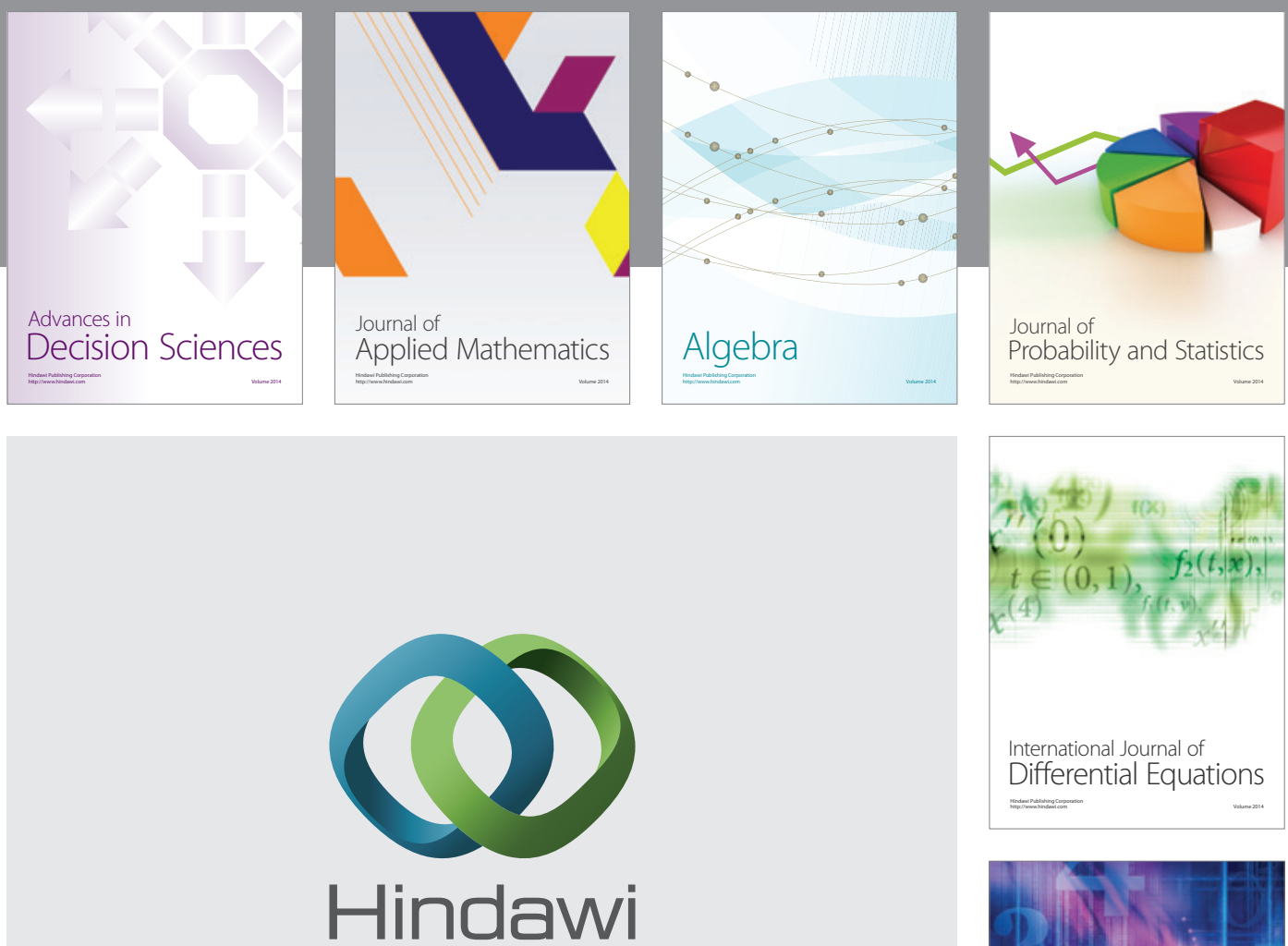

Submit your manuscripts at http://www.hindawi.com
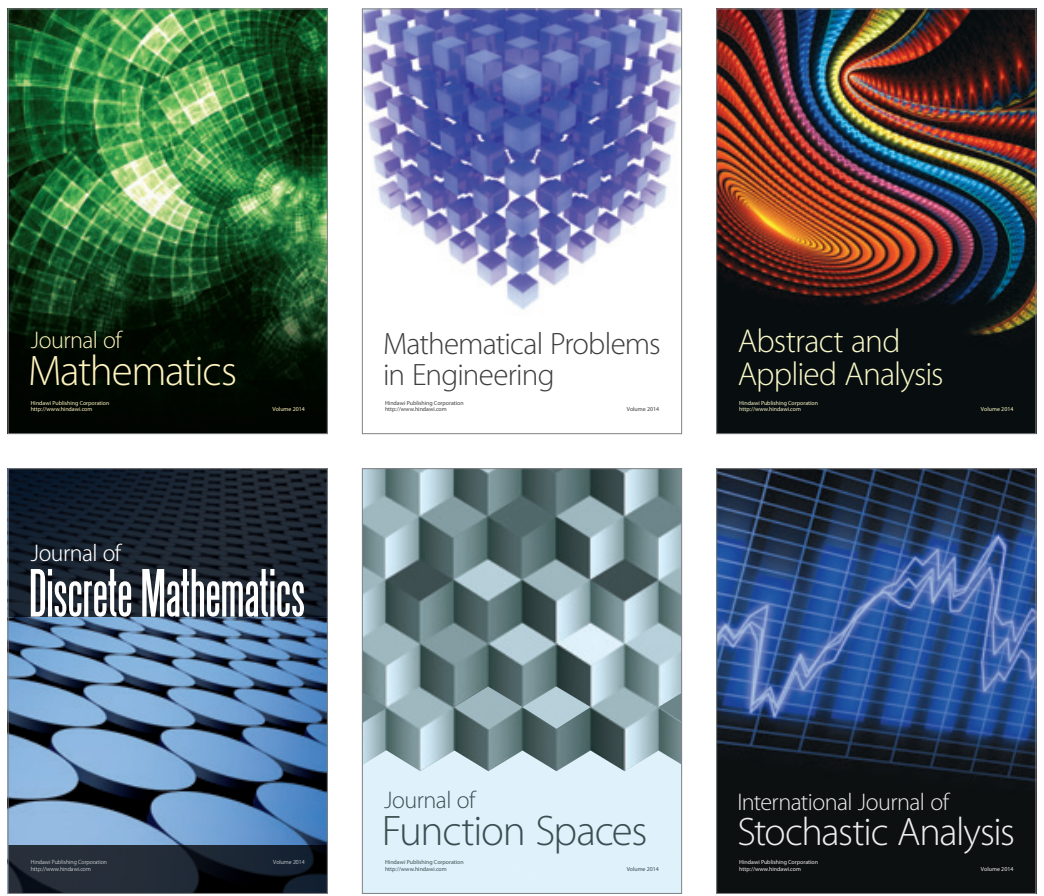

Journal of

Function Spaces

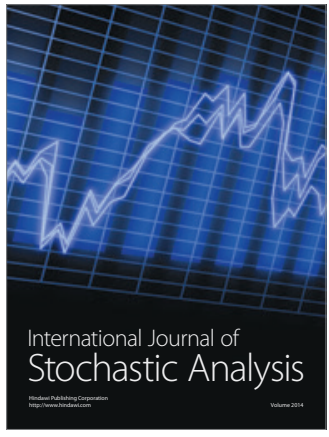

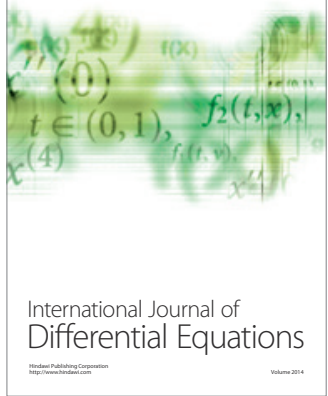
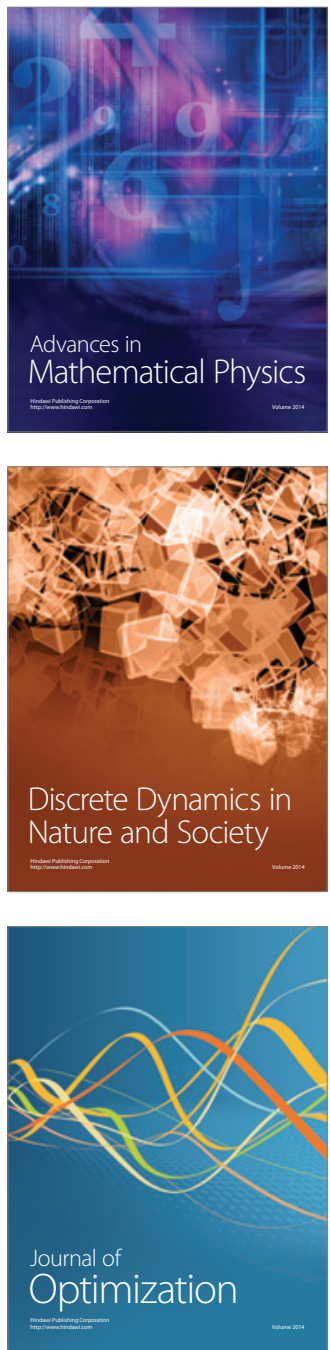